\title{
Assessment of Prescribing Pattern of Anti-Cancer Agents in Breast Cancer Patients at West Indian Oncology Hospital
}

\section{Avinash Khadela ${ }^{1 *}$, Bhavin Vyas ${ }^{1}$, Shrikant Joshi ${ }^{1}$, Sandesh Lodha ${ }^{1}$ and Tushar Bambharoliya ${ }^{2}$}

${ }^{1}$ Maliba Pharmacy College, Uka Tarsadia University, Surat, 394350, India

${ }^{2}$ North Carolina State University, NC USA

*Corresponding Author: Avinash Khadela, Maliba Pharmacy College, Uka Tarsadia University, Surat, 394350, India. E-mail: avinashkhadela@gmail.com
Received: July 24, 2020

Published: July 28, 2020

(C) All rights are reserved by Avinash

Khadela., et al.

DOI: $10.31080 /$ ASCB.2020.04.0247

\begin{abstract}
Background: Breast cancer (BC) is an emerging health issue not only for the developed nation but also for developing countries and very few literatures are available on drug utilization evaluation in patients with BC.

Objective: To examine the utilization pattern of anti-cancer agents in patients with $\mathrm{BC}$ in an Indian oncology setup.

Setting: The oncology department of Bharat Cancer Hospital in the western part of India.
\end{abstract}

Methods: In a descriptive-observational study treatment orders of patients on chemotherapy for BC were reviewed and the treatment pattern was assessed. Cancer patients who were above 18 years and are on chemotherapy along with supportive care medications were enrolled.

Main outcome measure: The most frequently used anti-neoplastic and supportive care agents, as a percentage of treatment.

Results: A total of 201 patients fulfilling inclusion criteria were enrolled in the study. The majority of the enrolled patients were in the age group of $50-69(54.7 \%)$ and females (100\%). The family history was found insignificant as a risk factor for the development of BC. The most common types of BC were ductal infiltrating (46.3\%), ductal invasive (35.8\%) and metastatic (9.0\%) and most of them were in stage III (53.2\%) and II (25.9\%). Amongst them, 18.4\% and 16.4\% of patients were suffering from oestrogen and progesterone positive tumors respectively whereas $6.5 \%$ was HER2 positive. Tamoxifen and anastrozole or letrozole were prescribed in oestrogen and progesterone positive BC respectively. Adjuvant chemotherapy (78.1\%) followed by Neo-adjuvant chemotherapy (16.9\%) were frequently prescribed. Amongst all anti-neoplastic agents prescribed cyclophosphamide (92.0\%) was highly prescribed followed by doxorubicin (79.1\%) and fluorouracil (37.8\%). Dexamethasone (61.2\%), ondansetron (50.8\%), palonosetron (32.8\%), pantoprazole (25.4\%), and peg-filgrastim (16.9\%) were commonly prescribed supportive care medications.

Conclusion: This study has shown that the majority of BC patients received alkylating agents followed by anthracycline derivatives and taxanes. Moreover, supportive care agents such as dexamethasone, ondansetron, and pantoprazole were administered in the majority of the patients along with peg-filgrastim or filgrastim to minimize chemotherapy induce toxicities.

Keywords: Breast Cancer; Chemotherapy; Prescribing patterns; Supportive Care

\section{Introduction}

Breast cancer $(\mathrm{BC})$ is an emerging health issue not only for the developed nation but also for developing countries. As per the world health organization (WHO) report, BC is one of the prominent health issues in women worldwide, involving 2.1 million women every year, and causing the maximum number of cancer-related deaths in women [1]. In India, BC has now surpassed the most commonly occurring cervical cancer and become a leading cause of cancer death [2]. In the year 2012, approximately 70,218 women died of BC in India that was the highest number of BC-related 
death across the globe that year. Moreover, the five years' survival of women suffering from BC in India is only $60 \%$ compare to the US where it is reported $89 \%$ [3]. The prime causes of high BC-related morbidity in Indian are lack of awareness regarding screening of $\mathrm{BC}$ and unavailability of appropriate medical care [4]. Therefore, it is important to understand current anti-neoplastic and supportive care utilization patterns.

The clinical factors such as tumor characteristics, clinical stage, and hormone receptor status were the most crucial factors in the selection of therapy. The management of advance stage $\mathrm{BC}$, was likely to initiate with surgery followed by adjuvant chemotherapy, neoadjuvant chemotherapy, and adjuvant hormonal therapy [5]. For the complete remission of malignant cells, chemotherapeutic regimens comprised of alkylating agents, anthracycline derivatives, taxanes and anti-metabolites either in combination or monotherapy have been used for the decades. Anthracycline based chemotherapy regimens are currently highly active in the management of $\mathrm{BC}$ and thus they are most prominently used as neo-adjuvant chemotherapeutic regimes [6]. In addition, alkylating agents and taxanes have shown improvement in survival of patients with advanced-stage $\mathrm{BC}$ and node-positive $\mathrm{BC}$ when prescribed as adjuvant therapy $[7,8]$.

The assessment of the prescribing pattern is a vital tool in ascertaining the current trend of medication utilization in various pathological conditions. In Indian setup, very few drug utilization evaluation studies in the field of oncology especially in BC have been published till now. Therefore, to get better insights into current management strategies or prescribing patterns among patients with BC in an Indian oncology setup, this study was performed to analyze the treatment pattern of anti-neoplastic agents in patients suffering from BC.

\section{Impact of findings on practice}

- To curb the incidence of advanced-stage breast cancer, appropriate screening tools, and related awareness should be provided to health care professionals and society.

- The usage of alkylating agents along with anthracycline derivatives are highly used chemotherapeutic regimes.

- Tamoxifen, anastrozole, and letrozole were used for the hormone-positive breast cancer patients to achieve better therapeutic yield and to avoid relapse.

\section{Materials and Methods}

Study design and site: It was a descriptive observational study conducted at Bharat Cancer Hospital, Surat, India. This is an oncology specialty hospital that gets patients mainly from the rural western region of India.

Inclusion criteria: All the newly diagnosed patients aged $>18$ years who were suffering from breast cancer and started with ei- ther adjuvant or neoadjuvant chemotherapy with or without radiation were included in the study.

Exclusion criteria: The patient receiving only radiation therapy for breast cancers and pregnant women were excluded from the study.

Sample size: A total of 201 patients were recruited in the study from February 2016 to February 2017.

\section{Study procedure}

An informed consent form was suitably designed to obtain consent from patients who volunteered for the study and fulfilled the study criteria. The patient was explained about the study and consent was obtained after they voluntarily agreed. Those patients were illiterate, the study was discussed with them and consent was obtained from the caretaker. The data were collected from the hospital's in-patients and ambulatory care departments. The information in each medication chart included the name, age, gender, family history, social habits, diet, height, weight, body surface area, address, clinical data such as diagnosis, past medication history, co-morbidities, allergy status, tumor size, stage of disease and therapeutic data such as name of the drug, dose, frequency, route, and duration of administration, concurrent medication(s), laboratory tests and results. Utilization evaluation of chemotherapeutic agents as adjuvant and neoadjuvant therapy was conducted on a qualitative basis. The utilization of anti-neoplastic agents from the medication charts of the enrolled patient were assessed as per the following criteria: (1) Segregation of medication charts as per the age, gender, co-morbidities, family history, social habits, anatomic sites of HNCs and types along with clinical stage. (2) Categorization of treatment protocol as per the clinical stage (3) Percentage of individual and therapeutic class wise anti-neoplastic and supportive care agents distribution.

\section{Statistical analysis}

The collected data of demographic and clinical variables were analyzed by Statistical Package for the Social Sciences (SPSS) using descriptive statistics namely frequencies and percentage.

\section{Results}

A total of 201 patients who were receiving anti-cancer agents as either adjuvant or neoadjuvant therapy for breast cancer (BC) were followed during 1 year of the study period.

$\mathrm{BC}$ was found more common $(\mathrm{n}=110,54.7 \%)$ among age group $50-69$ years followed by $36-49$ years $(n=55,27.4 \%), \leq 35$ years $(\mathrm{n}=26,13.0 \%)$. Only a few patients $(\mathrm{n}=10,5.0 \%)$ were age 70 and above. All the patients $(n=201,100 \%)$ followed during the study were female and only $49(24.4 \%)$ patients had a positive family history (first-degree relative) of BC. Most of the patients were mar- 
ried (85.0\%) and remaining were unmarried (5.0\%) and widows $(10.0 \%)$. Looking at the menstrual history of the patients, all the patients had their first menses during the age of 11 - 14 years and none of the patients had any known menstrual irregularity before the diagnosis of breast cancer. Menopausal status of the patients was found with almost equal distribution i.e. 96 and 105 out of 201 patients were in the pre-menopausal stage and post-menopausal stage respectively. Amongst 201 patients, 186 of them had their first pregnancy at the age of 30 or below. Among remaining patients, 10 of them were nulliparous and 5 of them had their first pregnancy at the age of 30-35 years. The majority (51.7\%) of the patients had the disease in the right breast, followed by left breast $(46.3 \%)$ and only $(2.0 \%)$ patients had the disease to both breasts.
Looking at the type of breast cancer, 93 patients had infiltrating carcinoma, 90 had invasive carcinoma and 18 patients had metastatic disease. Most of the patients had breast cancer in stage III A ( $n=53,26.4 \%)$, followed by stage II ( $n=52,25.9 \%)$ and stage III C ( $n=46,22.9 \%)$ (Table 1$)$. Most of the patients were tested to identify individuals with an endocrine responsive tumors. 175 out of 201 patients were tested, 37 of them were found to be positive with estrogen receptor and 33 of them were found to be positive for progesterone receptor. However, 26 patients were not tested for either estrogen receptor or progesterone receptor response. Amongst201 patients, 175 of them were also tested to assess response to the HER2 gene and 13 of them were found to be HER2 positive BC (Table 1).

\begin{tabular}{|c|c|c|c|}
\hline Demographic details & Number of patients $(n=201)(\%)$ & Demographic details & Number of patients $(n=201)(\%)$ \\
\hline \multicolumn{2}{|l|}{ Gender } & \multicolumn{2}{|c|}{ Tumor characteristics } \\
\hline Male & $00(00)$ & \multicolumn{2}{|c|}{ Involvement of breast } \\
\hline Female & $201(100)$ & Left & $93(46.3)$ \\
\hline \multicolumn{2}{|l|}{ Age groups } & Right & $104(51.7)$ \\
\hline$\leq 35$ & $26(12.9)$ & Both & $04(2.0)$ \\
\hline $36-49$ & $55(27.4)$ & \multicolumn{2}{|r|}{ Infiltrating } \\
\hline $50-69$ & $110(54.7)$ & Lobular & $00(00)$ \\
\hline$>70$ & $10(5.0)$ & Ductal & $93(46.3)$ \\
\hline \multicolumn{2}{|l|}{ Family history } & \multicolumn{2}{|r|}{ Invasive } \\
\hline Present & $49(24.4)$ & Lobular & $18(9.0)$ \\
\hline Absent & $152(75.6)$ & Ductal & $72(35.8)$ \\
\hline \multicolumn{2}{|l|}{ Marital status } & Metastatic & $18(9.0)$ \\
\hline Married & $171(85.0)$ & \multicolumn{2}{|c|}{ Age of first pregnancy } \\
\hline Unmarried & $10(5.0)$ & Nulliparous & $10(5.0)$ \\
\hline Separated & $00(00)$ & $\leq 30$ years & $186(92.5)$ \\
\hline Widow & $20(10.0)$ & 30-35 years & $5(2.5)$ \\
\hline \multicolumn{2}{|c|}{ Gender-specific patient features } & $36-39$ years & $0(00)$ \\
\hline Age of first menses & & $\geq 40$ years & $0(00)$ \\
\hline Before 10 years & $00(00)$ & \multicolumn{2}{|c|}{ Stages of Breast Cancers } \\
\hline $11-14$ years & $201(100)$ & Stage 0 & $12(6.0)$ \\
\hline \multicolumn{2}{|c|}{ Known menstrual abnormalities } & Stage I & $14(7.0)$ \\
\hline Yes & $00(00)$ & Stage II & $52(25.9)$ \\
\hline No & $201(100)$ & Stage IIIa & $53(26.4)$ \\
\hline Not Known & $00(00)$ & Stage IIIb & $08(4.0)$ \\
\hline \multicolumn{2}{|l|}{ Menopause status } & Stage IIIc & $46(22.9)$ \\
\hline Pre-menopause & $96(47.8)$ & Stage IV & $16(8.0)$ \\
\hline Post-menopause & $105(52.2)$ & & \\
\hline \multicolumn{4}{|c|}{ Hormone receptor status } \\
\hline Estrogen positive & 37 (18.4) & & \\
\hline Progesterone positive & $33(16.4)$ & & \\
\hline HER2 positive & $13(6.5)$ & & \\
\hline Triple negative & $92(45.8)$ & & \\
\hline Not tested & $26(12.9)$ & & \\
\hline
\end{tabular}

Table 1: Demographics, tumor characteristics and clinical stages related information. 
Majority patients (93.0\%) qualified for surgery after diagnosis and hence were operated soon after diagnosis and were placed on adjuvant chemotherapy. In few cases (16.9\%), the tumor was not operable as per the surgeon's opinion and hence they were prescribed with neoadjuvant therapy. Palliative care was suggested for $10(5.0 \%)$ patients due to the advanced stage of the disease. Based on the hormone receptor status, $84(41.8 \%)$ patients were prescribed with adjuvant endocrine therapy considering the patients menopausal status and $5(2.5 \%)$ patients were prescribed with targeted therapy. However, 68 (33.8) patients also received radiation therapy concomitantly along with either adjuvant or neoadjuvant therapy. Table 2 provides information about types of treatment modalities recommended for enrolled BC patients (Table 2).

\begin{tabular}{|l|c|}
\hline Treatment Regimen & $\begin{array}{c}\text { Number of patients } \\
\text { (n = 201) (\%) }\end{array}$ \\
\hline Adjuvant Chemotherapy & $157(78.1)$ \\
\hline Neo-adjuvant chemotherapy & $34(16.9)$ \\
\hline Adjuvant endocrine therapy & $84(41.8)$ \\
\hline Targeted therapy (trastuzumab) & $05(2.5)$ \\
\hline Palliative & $10(5.0)$ \\
\hline $\begin{array}{l}\text { Radiation (concomitant with } \\
\text { chemotherapy) }\end{array}$ & $68(33.8)$ \\
\hline Surgeries & $187(93.0)$ \\
\hline
\end{tabular}

Table 2: Number of patients receiving recommended treatment protocol for Breast Cancer.

Table 3 provides the list of chemotherapy regimens used for breast cancer patients according to their clinical stages.

\begin{tabular}{|l|c|c|}
\hline $\begin{array}{l}\text { Stage of the } \\
\text { disease }\end{array}$ & $\begin{array}{c}\text { Number of } \\
\text { Patients }\end{array}$ & Chemotherapy regimen prescribed (n = 201) \\
\hline Stage 0 & 12 & AC(8), CMF (4) \\
\hline Stage I & 14 & AC(2), FAC(6), TAC(2), EC(4) \\
\hline Stage II & 52 & AC(17), FAC(6), AC-PACL(2), FAC-DOCE(4), FEC(10), CMF(2), TA(2), TAC(4), PACL-A (6) \\
\hline Stage IIIA: & 53 & AC-PACL(2), FEC-DOCE(2), FEC(2), PACL-A (2) \\
\hline Stage IIIB & 08 & AC(20), FAC(10), AC-PACL(6), PACL-A (4), TAC(6) \\
\hline Stage IIIC & 46 & AC(2), FAC(6), TAC(4), DOCE (1), PACL (1), FEC(2) \\
\hline Stage IV & 16 & \multicolumn{2}{|c|}{} \\
\hline $\begin{array}{l}\text { Chemotherapy abbreviation } \\
\text { AC: Doxorubicin, Cyclophosphamide. CMF: Cyclophosphamide, Methotrexate, Fluorouracil. FAC: Fluorouracil, Doxorubi- } \\
\text { cin, Cyclophosphamide. FEC: Fluorouracil, Epirubicin, Cyclophosphamide. PACL: Paclitaxel. TAC: Docetaxel, Doxorubicin, } \\
\text { Cyclophosphamide. PACL A: Paclitaxel, Doxorubicin. CMF: Cyclophosphamide, Methotrexate, Fluorouracil. EC: Epirubi- } \\
\text { cin, Cyclophosphamide. DOCE: Docetaxel. TA: Docetaxel, Doxorubicin. }\end{array}$ \\
\hline
\end{tabular}

Table 3: Stages of the breast cancer and chemotherapy regimen prescribed.

Most of the patients had BC in stage III A ( $n=53,26.4 \%)$ and were given with different combinations of chemotherapy regimens, followed by stage II $(n=52,25.9 \%)$ and stage III C ( $n=46,22.9 \%)$. The majority of patients $(69 \%)$ were prescribed with the AC regimen followed by FAC (34\%), FEC (20\%). The single-drug treatment (2\%) was given only for palliative purpose (Figure 1).

A total of 7 potential chemotherapeutic agents were used in 201 enrolled patients. Amongst all anticancer agents used cyclophosphamide $(92.0 \%)$ was the most prescribed drug followed by doxorubicin (79.1\%) and fluorouracil (37.8\%), while methotrexate $(5.0 \%)$ was least used. Table 4 provides the prescribing patterns of individual anti-neoplastic and supportive care agents. Supportive care agents include anti-emetics, gastrointestinal drugs, and GCSF. Moreover, all the pre-menopausal patients with endocrine responsive tumors were prescribed with tamoxifen oral tablet (47.8\%) and post-menopausal patients were prescribed with anastrozole
(29.4\%) and letrozole (22.9\%) oral tablet respectively. The patients with HER2 expressive gene were prescribed with trastuzumab (2.5\%) (Table 4).

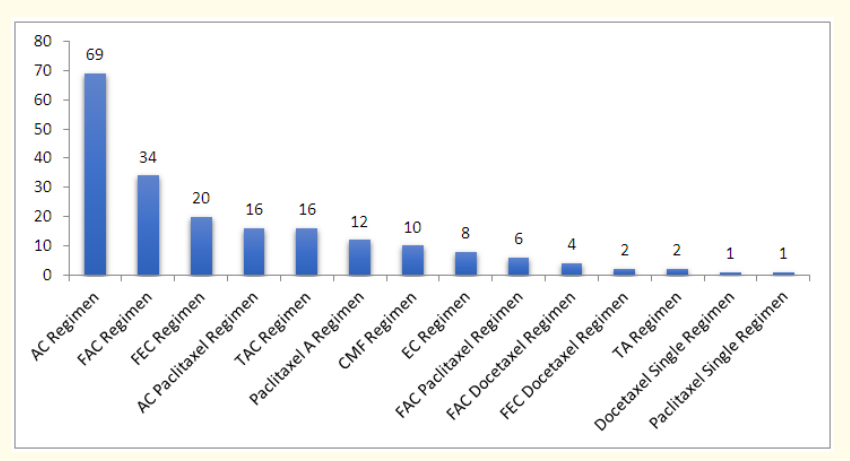

Figure 1: Adjuvant and Neo-adjuvant chemotherapy used for Breast cancer. 


\begin{tabular}{|l|c|c|c|}
\hline $\begin{array}{l}\text { Therapeutic/chemical classes of } \\
\text { medication }\end{array}$ & Name of medicine & ATC code & $\begin{array}{c}\text { Number of pts prescribed } \\
\text { (n = 201) (\%) }\end{array}$ \\
\hline Anti-neoplastic agents & Cyclophosphamide & L01AA01 & $185(92.0)$ \\
\hline Alkylating agents & Doxorubicin & L01DB01 & $159(79.1)$ \\
\hline \multirow{2}{*}{ Anthracycline derivatives } & Epirubicin & L01DB03 & $30(14.9)$ \\
\hline \multirow{2}{*}{ Taxanes } & Paclitaxel & L01CD01 & $35(17.4)$ \\
\cline { 2 - 4 } & Docetaxel & L01CD02 & $25(12.4)$ \\
\hline \multirow{2}{*}{ Antimetabolites } & Fluorouracil & L01BC02 & $76(37.8)$ \\
\hline \multirow{2}{*}{ Supportive care agents } & Methotrexate & L04AX03 & $10(5.0)$ \\
\hline \multirow{2}{*}{ Antiemetics } & \multicolumn{2}{|c|}{} \\
\cline { 2 - 4 } & Palonosetron & A04AA05 & $72(35.8)$ \\
\cline { 2 - 4 } & Ondansetron & A04AA01 & $102(50.8)$ \\
\cline { 2 - 4 } & Dexamethasone & A01AC02 & $123(61.2)$ \\
\cline { 2 - 4 } & Metoclopramide & A03FA & $06(3.0)$ \\
\hline Gastrointestinal drugs & Pantoprazole & A02BC02 & $51(25.4)$ \\
\cline { 2 - 4 } & Ranitidine & A02BA02 & $37(18.4)$ \\
\hline Granulocyte-colony stimulating factors & Filgrastim & L03AA02 & $17(8.5)$ \\
\cline { 2 - 4 } (GCSFs) & Peg-Filgrastim & L03AA13 & $34(16.9)$ \\
\hline Endocrine therapy & Tamoxifen & L02BA01 & $96(47.8)$ \\
\cline { 2 - 4 } & Anastrozole & L02BG03 & $59(29.4)$ \\
\hline \multirow{4}{*}{ Targeted therapy } & Letrozole & L02BG04 & $46(22.9)$ \\
\hline & Trastuzumab & L01XC03 & $05(2.5)$ \\
\hline
\end{tabular}

Table 4: Prescription pattern of anticancer and supportive care agents.

\section{Discussion}

Breast cancer (BC) remains a major health burden in the developing world. Chemotherapy, over the years, has been used in an attempt to reduce the morbidity rates, recurrence rates and increase the survival rates of BC patients. This, however, has resulted in its imprudent use and associated consequences of increased resistance to chemotherapy treatment, unnecessary adverse reactions and inappropriate management of patients [9]. This work is focussed on assessing the utilization patterns of chemotherapy drugs used in the management of BC in an oncology setup.

In this study, we tried to relate the risk of patient characteristics (age, gender, family history, early menarche, late menopause, pregnancy, and marital status) and the development of BC.

According to the study conducted by Chopra B., et al. and Sandhu D., et al, the incidence of BC in Indian women has been increased amongst the age group 45-54 years which were a decade earlier than western women. We also observed that majority of our study patients were in the age group 50-69 years $[10,11]$. Concerning the risk of $\mathrm{BC}$ and family history, as per the cohort study conducted by Brewer HR., et al. only 5\% have reported a low rate of a familial pattern of BC in Indian patients while in our study we observed that 49 (24.4\%) patients had positive family history of BC [12]. The information related to the menstrual status of women may help in predicting the risk of $\mathrm{BC}$ as it is considered as the most prominent risk factor for the development of BC. Menstrual history such as information regarding early menarche, late menopause, and nulliparity have a significant role in predicting the risk of BC [13]. In the cohort study conducted by Bodicoat., et al. has shown that women with a history of early menarche and the study conducted by Singletary SE shown that women who did not reach menopause until age 55 or later had a high risk of developing BC. The early menarche and attainment of delayed menopause increase the lifetime risk of estrogen exposure which is considered as one of the potential risk factors for the BC. However, in our study, none of the enrolled patients had a history of early menarche and delayed menopause [13,14].

The clinical staging of $\mathrm{BC}$ plays a vital role in predicting treatment strategies and prognosis. Agarwal G., et al. in their study have revealed that the detection of $\mathrm{BC}$ in advanced stage may be attributed to a lack of awareness about the screening of disease or periodic examination by health care specialists. In our study, we observed 
that most of the patients were found in clinically advanced stage i.e. stage III (53.3\%) [15]. These high numbers were mainly due to delayed oncologist consultation and consulting local physicians for longer duration irrespective of treatment failures. Hormone status is an important tool for BC differentiation and a vital predictor of response to hormonal therapy and overall survival [16]. According to the meta-analysis conducted by Sandhu GS., et al. the prevalence of triple-negative BC in Indian women was significantly high compare to western. Similarly, in our study, we observed the maximum patients (approximately more than 70\%) had the triplenegative hormonal status [17]. The management of BC is comprised of surgical approach, neoadjuvant chemotherapy, adjuvant chemotherapy and in case of very advanced stage palliative care with or without radiation therapy [5]. Poole CJ., et al. in their study revealed that tumor resection followed by adjuvant chemotherapy in the early stages of $\mathrm{BC}$ reduces the risk of disease relapse and a preferable managing strategy. Similar findings were observed in our study as the majority of patients (78.1\%) were prescribed with adjuvant chemotherapy followed by neoadjuvant chemotherapy (16.9\%) [18]. The decision regarding tumor resection is usually taken by a surgical oncologist in the clinical conditions when tumors are surgically removable whereas the medication-related decisions are usually taken by medical oncologists after considering the surgical oncologist opinion. Considering the role of adjuvant radiation therapy along with chemotherapy, Joshi SC., et al. in their study revealed that breast irradiation therapy could significantly reduce the risk of local recurrence and help in improving overall survival. In our study, we found that $33.8 \%$ of patients had been recommended with radiation therapy along with chemotherapy as suggested by the radiation oncologist [19]. The selection of appropriate anti-neoplastic agents is very crucial to get maximum safety and efficacy while treating the $\mathrm{BC}$ patients. According to the study conducted by Campbell HE., et al. alkylating agents, anthracycline derivatives and taxanes were the most effective anti-cancer agents in the management of BC. We found similar results in our study as most of the study patients have been prescribed with Adriamycin and cyclophosphamide regimen $(n=69)$ [20].

The hormonal therapy in the eradication of BC plays a pivotal role as it is well established that estrogen and progesterone are major risk factors along with the HER2 gene for breast malignancy. Therefore, it is essential to identify and treat the hormone receptor sensitive tumors by prescribing rational adjuvant hormonal therapy. In this study, we found $18.4 \%$ and $16.4 \%$ of patients had estrogen and progesterone positive $\mathrm{BC}$ respectively [21]. Amongst all the enrolled patients, 26 patients the hormone receptor status was unknown as they were not tested. These patients were either underwent for lumpectomy or receiving palliative care and in these both cases, the hormonal testing result would be insignifi- cant. However, in our study, those patients whose hormone status was not known were prescribed with endocrine therapy i.e. 11 and 8 patients received estrogen and progesterone respectively along with chemotherapy. Besides, HER2 is also an important risk factor for the development of $\mathrm{BC}$ as its expression is an individual prognostic factor for predicting the aggressive behavior of the tumor as well as the benefit from adjuvant therapy [22]. Clinical trials have shown that the relative risk of recurrence is decreased by $50 \%$ and prolong survival when trastuzumab is added to the adjuvant chemotherapy regimen in HER2-positive women. In our study, 13 patients were suffering from HER2 positive BC however, only 5 patients out of them were prescribed with trastuzumab [23]. The probable reason behind not prescribing trastuzumab in eligible patients was financial constrain as trastuzumab is very expensive.

Menopause status plays a vital role in the selection of appropriate hormonal therapy. Selective estrogen receptor modulator such as tamoxifen has a significant effect in premenopausal women whereas anastrozole and letrozole have higher efficacy in postmenopausal women suffering from BC. In our study, we observed that 96 pre-menopausal and 105 post-menopausal women received tamoxifen and anastrozole and letrozole respectively [16,24]. Supportive care agents such as dexamethasone (100\%), palonosetron (100\%), pantoprazole $(25.4 \%)$ and ranitidine $(18.4 \%)$ were highly prescribed in the study conducted by Ramalakshmi., et al. to prevent chemotherapy-induced toxicities. In our study, we observed that dexamethasone $(61.2 \%)$, ondansetron $(50.8 \%)$, palonosetron (35.8\%), pantoprazole (25.4\%) and ranitidine (18.4\%) were highly used along with pegfilgrastim (16.9\%) and filgrastim (8.5\%) $[25,26]$.

The study has certain limitations such as we did not check the rationality of prescription using WHO indicators and due to the unavailability of the accurate prevalence of BC in India we did not calculate the exact sample size. Moreover, hospital formulary and limited government funding do not allow the clinicians to adopt the national comprehensive cancer network (NCCN) guidelines or Australian cancer institute New South Wales sites eviQ guidelines and therefore we did not compare our prescribing pattern with these guidelines.

\section{Conclusion}

The study has shown that the prevalence of BC was high in patients having age more than 50 years. Family history as a contributing factor in the development of BC was insignificant as it was absent in all the enrolled patients. The ductal infiltrating carcinoma of breast was more common compare to other types of breast cancer namely ductal invasive and metastatic. Patients were diagnosed in an advanced stage of $\mathrm{BC}$ mainly because of the negligence of symp- 
toms and education regarding the screening of breast tumors. The prominent risk factors for the development of BC is oestrogen, progesterone, and HER2 gene expression, however, the study has shown a high prevalence of triple-negative BC. For the management of hormone-positive breast cancer tamoxifen, anastrozole and letrozole were prescribed to minimize relapse and disease progression. The treatment approach was comprised of adjuvant and neoadjuvant chemotherapy based on the clinical stage. The commonly used antineoplastic agents were cyclophosphamide, doxorubicin, and 5-fluorouracil and supportive medicines such as dexamethasone, 5-hydroxytryptamine (5-HT3) antagonist, pantoprazole and peg-filgrastim.

\section{Acknowledgments}

The author wholeheartedly wishes to acknowledge all the concern physicians and nursing staff of Bharat Cancer Hospital, Surat who have supported for the smooth conductance of study.

\section{Funding}

The study was not funded.

\section{Conflict of Interests}

All the author(s) have declared no conflict of interest.

\section{Ethics Approval}

The study was ethically approved by the Institutional ethics committee of Maliba Pharmacy College, Bardoli. Reference number: MPC/HREC/01/2015-16; dated: 22/12/2015.

\section{Informed Patient Consent}

The purpose and protocols of the study were explained to patients, and written consent was obtained.

\section{Author Contributions}

AK designed and conducted the study, collected data and wrote the manuscript. All others have designed and performed the analysis of collected data.

\section{Bibliography}

1. Cancer. World health organization (2020).

2. Malvia S., et al. "Epidemiology of breast cancer in Indian women". Asia-Pacific Journal of Clinical Oncology 13 (2017): 289295.

3. Statistics of breast cancer in India (2020).

4. Tfayli A., et al. "Breast cancer in low- and middle-income countries: An emerging and challenging epidemic". Journal of Oncology 2010 (2010): 1-5.

5. Giordano SH., et al. "Breast cancer treatment guidelines in older women". Journal of Oncology 23(4) (2005): 783-791.
6. Smith IC., et al. "Neoadjuvant chemotherapy in breast cancer: significantly enhanced response with docetaxel". Journal of Oncology 20.6 (2002): 1456-1466.

7. Rodenhuis S., et al. "Efficacy of high-dose alkylating chemotherapy in HER2/neu-negative breast cancer". Annuals of Oncology 17.4 (2006): 588-596.

8. Walshe JM., et al. "Amenorrhea in premenopausal women after adjuvant chemotherapy for breast cancer". Journal of Oncology 24.36 (2006): 5769-5779.

9. Ghoncheh M., et al. "Incidence and Mortality and Epidemiology of Breast Cancer in the World". Asian Pacific Journal of Cancer Prevention 17.S3 (2016): 43-46.

10. Chopra B., et al. "Age shift: breast cancer is occurring in younger age groups-is it true?" Clinical Cancer Investigation Journal 3 (2014): 526-529.

11. Sandhu D., et al. "Profile of breast cancer patients at a tertiary care hospital in north India". Indian Journal of Cancer 47 (2010): 16-22.

12. Brewer HR., et al. "Family history and risk of breast cancer: an analysis accounting for family structure". Breast Cancer Research and Treatment 165.1 (2017): 193-200.

13. Singletary SE. "Rating the Risk Factors for Breast Cancer". Annuals of Surgery 237.4 (2003): 474-482.

14. Bodicoat DH., et al. "Timing of pubertal stages and breast cancer risk: the Breakthrough Generations Study". Breast Cancer Research 16.1 (2014): R18.

15. Agarwal G and Ramakant P. "Breast Cancer Care in India: The Current Scenario and the Challenges for the Future". Breast Care (Basel) 3.1 (2008): 21-27.

16. Desai SB., et al. "Hormone receptor status of breast cancer in India: a study of 798 tumours”. Breast 9.5 (2000): 267-270.

17. Sandhu GS., et al. "Prevalence of Triple-Negative Breast Cancer in India: Systematic Review and Meta-Analysis". The Journal of Global Oncology 2.6 (2016): 412-421.

18. Poole CJ., et al. "Epirubicin and cyclophosphamide, methotrexate, and fluorouracil as adjuvant therapy for early breast cancer". The New England Journal of Medicine 355.18 (2006): 1851-1862.

19. Joshi SC., et al. "Role of radiotherapy in early breast cancer: an overview". International Journal of Health Sciences (Qassim). 1.2 (2007): 259-264.

20. Campbell HE., et al. "The cost-effectiveness of adjuvant chemotherapy for early breast cancer: A comparison of no chemotherapy and first, second, and third generation regimens for patients with differing prognoses". European Journal of Cancer 47.17 (2011): 2517-2530. 
21. Jones KL and Buzdar AU. "A review of adjuvant hormonal therapy in breast cancer". Endocrine-Related Cancer 11.3 (2004): 391-406.

22. Iqbal $\mathrm{N}$ and Iqbal N. "Human Epidermal Growth Factor Receptor 2 (HER2) in Cancers: Overexpression and Therapeutic Implications". Molecular Biology International 2014 (2014): 852748.

23. Carpenter RL and Lo HW. "Regulation of Apoptosis by HER2 in Breast Cancer". Journal of Carcinogenesis and Mutagenesis 2013 (2013): 003.

24. Mouridsen HT and Robert NJ. "The role of aromatase inhibitors as adjuvant therapy for early breast cancer in postmenopausal women". European Journal of Cancer 41.12 (2005): 1678-1689.

25. Ramalakshmi S., et al. "A study on prescribing trends of supportive care drugs used in cancer chemotherapy in a tertiary care teaching hospital". IJOPP 6 (2013): 36-39.

26. Khadela A and Vyas B. "Assessment of the utilization pattern of chemotherapy agents in patients with head and neck cancer in an oncology hospital". Drugs and Therapy Perspectives 36.7 (2020): 303-309.

\section{Assets from publication with us}

- Prompt Acknowledgement after receiving the article

- Thorough Double blinded peer review

- Rapid Publication

- Issue of Publication Certificate

- High visibility of your Published work

Website: www.actascientific.com/

Submit Article: www.actascientific.com/submission.php

Email us: editor@actascientific.com

Contact us: +919182824667 